Service social

\title{
Marguerite B. - Une jeune fille en maison de correction, par Béatrice Koeppel, Paris, Hachette, 1987, 238 pages.
}

\section{Lise Binet}

Volume 35, numéro 3, 1986

Les jeunes et le travail social

URI : https://id.erudit.org/iderudit/706330ar

DOI : https://doi.org/10.7202/706330ar

Aller au sommaire du numéro

Éditeur(s)

École de service social de l'Université Laval

ISSN

1708-1734 (numérique)

Découvrir la revue

Citer ce compte rendu

Binet, L. (1986). Compte rendu de [Marguerite B. - Une jeune fille en maison de correction, par Béatrice Koeppel, Paris, Hachette, 1987, 238 pages.] Service social, 35(3), 485-487. https://doi.org/10.7202/706330ar d'utilisation que vous pouvez consulter en ligne.

https://apropos.erudit.org/fr/usagers/politique-dutilisation/ 
D'autres résultats donnent à réfléchir et méritent que le questionnement soit poussé plus loin, particulièrement en ce qui a trait au dépôt des évaluations sociales, aux requêtes de placement, dans le cas des adolescents qui présentent des problèmes de comportement, à la fonction confirmative plutôt qu'adjudicative du Tribunal et à la propension des parents et des enfants à consentir aux requêtes du D.P.J., sans nécessairement négocier, débattre et argumenter.

II y a là un phénomène à scruter de près, qui révèle ou le désengagement parental ou une certaine forme de défaitisme des parents et des enfants qui perçoivent l'inutilité de batailler contre les puissantes machines sociales et judiciaires. C'est là une interrogation qui doit rester présente jusqu'à ce qu'on ait acquis la certitude que nos institutions administratives et judiciaires offrent et facilitent vraiment la possibilité de se faire entendre, dans un processus qui n'aurait pas qu'une trompeuse apparence démocratique.

Finalement, pour ce qui est relatif à la portée de l'ouvrage, certaines données apparaissent, à première vue, généralisables à la pratique habituelle en contexte québécois. Par contre, la prudence s'impose. Cette étude ne vaut que pour l'année de référence, et les observations faites ne s'appliquent qu'à une partie des décisions judiciaires en matière de protection. On se rappellera, qu'entre autres, les requêtes en révision et en prolongation étaient exclues de la recherche. D'autre part, on peut concevoir que les pratiques sociale et judiciaire d'autres régions puissent différer à plusieurs égards. Qu'il suffise ici de mentionner qu'à Montréal, le volume de cas n'est pas le même, les évaluations sociales et autres expertises font fréquemment partie de la preuve documentaire et les jugements rendus prévoient presque toujours la mesure de suivi social, même lorsqu'il y a ordonnance de placements de divers types.

Pour conclure, disons que l'initiative est intéressante et qu'il serait souhaitable qu'il y ait des suites à cette première percée. Qu'on me permette enfin, pointe amicale aux juristes (éditeur ou auteur), de signaler qu'ils ont omis d'effectuer, à la Bibliothèque nationale du Québec, l'enregistrement et le dépôt légal prescrits par la Loi.

Direction de la protection de la jeunesse,

Jean GosseuIN

Centre de services sociaux du Montréal métropolitain.

Marguerite B. - Une jeune fille en maison de correction, par Béatrice KoEPPEL, Paris, Hachette, 1987, 238 pages.

Marguerite B., 20 ans, internée depuis trois ans à Cadillac, établissement correctionnel français pour mineures, se suicide par pendaison un jour d'octobre 1950. C'est ce dossier que Béatrice Koeppel ouvre, plus de 30 ans après la mort de celle qui avait battu et volé sa mère infirme, trahi la confiance de sa famille bienfaitrice, se mutilait et aurait été encline à la simulation et au mensonge. 
Se concentrant sur le cas Marguerite, l'auteure cherche, à l'aide des pièces du dossier, dont les écrits n'ont pourtant aucune prétention théorique, à mettre en lumière les discours dominants tenus sur les femmes, les jeunes et la délinquance, qui marquent la conception de la justice des mineures de l'époque. Au-delà d'un drame individuel, c'est l'interprétation donnée par les discours institutionnels qui intéresse, d'autant plus que ceux qui se côtoient, dans la période charnière des années 1950, révèlent un changement de conception de la délinquance juvénile et de la philosophie rééducative.

C'est au cours de cette période qu'apparaît la notion d'inadaptation sociale, qui s'écarte des idéologies répressives. En effet, jusque-là, on s'appliquait à réprimer les comportements malsains et pervers des mineures, comportements qui n'avaient d'autres causes identifiées que le déterminisme biologique. Avec Lombroso, les délinquantes sont désignées comme des jeunes filles avec le vice dans la peau et qu'il faut punir. Porte-parole d'un savoir alors considéré irréfutable, il véhicule de plus la certitude que les femmes sont naturellement portées à travestir la vérité.

L'auteure retrouve dans le dossier de Marguerite trace de discours dont la logique permet de traduire ses actes en termes de simulation. Marguerite, mythomane, se voit nier la vérité de sa souffrance et l'authenticité de ses gestes et même de sa mort puisque, écrit-on, elle serait accidentelle, n'étant pas véritablement souhaitée. Cette interprétation, héritée du XIXe siècle, accompagne celle développée par Lagache pour qui les comportements anti-sociaux s'expliquent par des conflits manifestés entre les exigences de l'adaptation sociale et les désirs personnels du mineur. L'auteure y voit d'ailleurs là l'énoncé qui permettra, dans les années 1960, de systématiser la procédure d'enquête sociale où les psychologues doivent "découvrir le système des mobiles irréfléchis et inconscients qui soustendent l'acte délictueux» (p. 80).

L'investigation psychosociale, qui marque alors le pas sur la répression des enfants « malades», attire maintenant l'attention sur la famille dissociée à laquelle on raccroche l'histoire des mineurs déviants à qui il faut imposer de nouvelles normes éducatives. Ces conceptions se retrouvent aussi au niveau des établissements d'éducation surveillée. La fin des années 1930 fut marquée par de nombreux scandales sur les bagnes d'enfants qui suscitèrent des réformes au cours de la décennie suivante. Au scoutisme, succède le projet rééducatif où, pour les jeunes, « une image sociale sublimée doit balayer la force des premiers investissements " (p. 155) c'est-à-dire, réinvestir dans une éducation modèle qui coupe avec la mauvaise famille.

Cependant, et c'est là l'essentiel de la thèse de l'auteure, réprimée ou rééduquée, il y a toujours en toile de fond la négation du sujet, dans ce cas-ci féminin et jeune. Elle rappelle les conceptions d'avant-garde développées par les neuro-psychiatres de l'École de Genève et du mouvement psychanalytique français qui rompent avec l'étiologie biologique de la maladie mentale et reprennent, dans l'œuvre de Freud, sa conception de l'hystérie. II n'y aurait pas d'équivoque : Freud a infirmé l'hypothèse qui associe l'hystérie à la simulation et " dénie au fantasme toute valeur de vérité en qualifiant de simulation les artifices 
de l'imagination ". Dans cette perspective, de dire l'auteure, " seuls comptent les actes et les conduites, jamais les significations " (p. 193).

Dans le secteur de la justice des mineurs, ce sera toujours la simulation qui primera : on ne croit pas les femmes et les enfants. Pourtant, conclut l'auteure : "Reconnaître que l'autre souffre, qu'il est profondément triste, plus encore qu'il aspire à mourir, c'est admettre l'authenticité de sa douleur et donc, lui donner une chance de parler, d'être entendu pour sa vraie peine, lui permettre de vivre. Refuser a priori cette dimension revient à lui ôter le droit d'une expression libre." (P. 203.)

Béatrice Koeppel a réussi à écrire un livre qui se lit comme un roman, tout en «brassant » de grosses théories qui la rapprochent des analyses de discours de Foucault et de l'anti-psychiatrie. Aux informations provenant du dossier de Marguerite, elle ajoute celles contenues dans les entrevues menées auprès de personnes qui l'ont connue. À ces diverses voix qui se font entendre, s'ajoute celle de Marguerite qui s'exprime dans les lettres qu'elle adressait à la Directrice de l'établissement et que l'auteure a reproduites sans aucun commentaire, fidèle à sa démarche de fournir une biographie institutionnelle et non personnelle. Ce sont des discours qui dominent la justice des mineures qu'elle rend compte et non de celui du sujet. À cela, s'ajoutent les quelques incursions personnelles de l'auteure dans son propre discours "scientifique".

Bien que rendant compte d'une réalité française, et que l'on soit ou non en accord avec le recours à la notion d'hystérie pour formuler une critique, ce livre ouvre des perspectives intéressantes et stimulantes pour questionner notre propre système d'aide à la jeunesse et le traitement fait à la déviance féminine puisque, dans ces domaines, les perspectives convergent sur de nombreux points.

Centre de services sociaux de Québec.

Lise BINET

Qualité de vie et drogues - Place aux jeunes, par l'Association des intervenants en toxicomanie du Québec, Chicoutimi, Gaëtan Morin, 1986, 262 pages.

Ce volume constitue les Actes du quatorzième colloque de l'Association des intervenants en toxicomanie du Québec, qui s'est tenu à Longueuil en mai 1985, pendant l'Année internationale de la jeunesse. Dans son organisation, l'ouvrage suit les différentes catégories de présentations annoncées dans le programme.

On trouve d'abord les textes des trois conférences principales. Jeanned'Arc Vaillant, alors sous-ministre adjointe, présente le projet d'orientations du Ministère des affaires sociales (Québec) à l'égard de l'usage et de l'abus des psychotropes. Roland Reumond aborde différentes stratégies d'éducation et de prévention développées en Belgique. Enfin, Marie-Andrée Bertrand questionne 